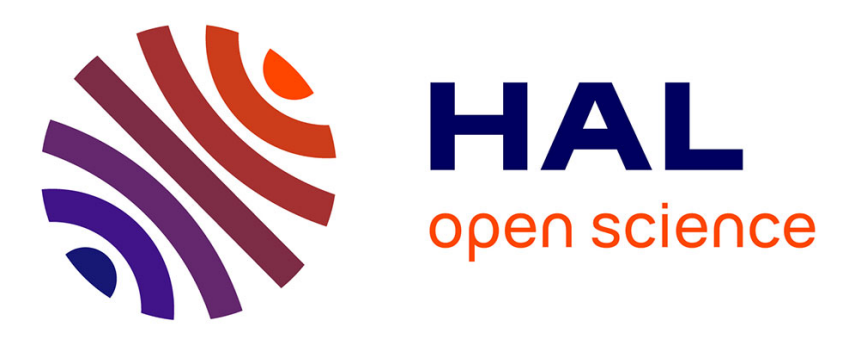

\title{
Solvent screening methodology for in situ ABE extractive fermentation.
}

Helena Gonzalez-Penas, T. A. Lu-Chau, Manuel Moreira, J. M. Lema

\section{To cite this version:}

Helena Gonzalez-Penas, T. A. Lu-Chau, Manuel Moreira, J. M. Lema. Solvent screening methodology for in situ ABE extractive fermentation.. Applied Microbiology and Biotechnology, 2014, 98 (13), pp.5915-5924. 10.1007/s00253-014-5634-6 . hal-01025657

\section{HAL Id: hal-01025657 \\ https://hal-ifp.archives-ouvertes.fr/hal-01025657}

Submitted on 18 Jul 2014

HAL is a multi-disciplinary open access archive for the deposit and dissemination of scientific research documents, whether they are published or not. The documents may come from teaching and research institutions in France or abroad, or from public or private research centers.
L'archive ouverte pluridisciplinaire HAL, est destinée au dépôt et à la diffusion de documents scientifiques de niveau recherche, publiés ou non, émanant des établissements d'enseignement et de recherche français ou étrangers, des laboratoires publics ou privés. 


\title{
Solvent screening methodology for in situ ABE extractive fermentation
}

\author{
$\underline{\text { H. González-Peñas }}^{1}$, T.A. Lu-Chau², M.T. Moreira ${ }^{3}$, J.M. Lema ${ }^{3}$ \\ ${ }^{1}$ IFP Energies Nouvelles, Rond-point de l'échangeur de Solaize BP 3, 69360 Solaize, France. \\ ${ }^{2}$ Department of Chemical Engineering, Institute of Technology, University of Santiago de \\ Compostela, 15782, Santiago de Compostela, Spain; \\ ${ }^{3}$ Department of Chemical Engineering, School of Engineering, University of Santiago de Compostela, \\ 15782, Santiago de Compostela, Spain. \\ E-mail address: thelmo.lu@usc.es
}

\begin{abstract}
A systematic solvent screening methodology for the in situ liquid extraction of products from Acetone-Butanol-Ethanol fermentation was developed in this study. on the basis of theoretical considerations, sixteen compounds from different chemical families were selected and experimentally evaluated for their extraction capabilities in a real $A B E$ fermentation broth system. The best potential solvents were also tested for their biocompatibility towards Clostridium acetobutylicum. Differences in substrate consumption and total butanol production referred to solvent-free fermentations were quantified for each solvent. It was observed that a) the excess of glucose consumption does not lead systematically to the same amount of overproduction of butanol with the biocompatible solvents and b) product yield was enhanced in the presence of solvents having higher affinity for butanol and butyric acid. Applying this methodology, it was found that the solvent that presented the best extracting characteristics and maintained biocompatibility with $C$. acetobutylicum was the Guerbet alcohol 2-butyl-1-octanol.
\end{abstract}

Key words: Liquid-liquid extraction, Clostridium acetobutylicum, partition coefficient, selectivity, biocompatibility 


\section{Introduction}

The incessant fluctuations in oil prices and the depletion of fossil resources have generated renewal interest and efforts towards the synthesis of fuels and chemicals from renewable raw materials. Butanol presents some advantages over ethanol in terms of lower hygroscopicity and higher energy density. Furthermore, butanol can also be used as a feedstock in petrochemical industry. Therefore, increasing attention has been recently paid to biotechnological production of butanol. Acetone-Butanol-Ethanol (ABE) fermentation was the second largest industrial scale fermentation before the rise of petroleum refinery based industry. However, ABE fermentation is subject to strong inhibition by products (Jones \& Woods 1986), which leads to low final product concentration, high recovery costs and large volumes of wastewater generation.

Over the past three decades, extensive research has been focused on finding solutions to overcome the butanol toxicity problem. These include: (a) genetically modification of solventogenic Clostridia to enhance butanol toxicity tolerance, (b) metabolic engineering of butanol biosynthesis in well characterized microorganisms (Ezeji et al 2010), and (c) integration of the fermentation process with in situ primary separation systems. The later approach allows the recovery of butanol as fast as it is produced, and thus it would keep the butanol concentration under the inhibition threshold. Other alternatives that have been explored are adsorption, gas stripping, liquid-liquid extraction, pervaporation, etc. (Oudshoorn et al 2009, Leland 2008, Woodley et al 2008, Qhreshi et al 2005).

It has been reported that in situ solvent extraction presents substantial energy savings over distillation for butanol recovery from highly diluted broths (Qhreshi et al 2005). Lower requirements of energy lead to increased energy ratio for butanol production and globally reduced operating costs.

The solvent choice is one of the most important steps of the development of in situ extractive fermentations. Several works have focused on the research of suitable solvents for butanol extractive fermentations (Dadgar \& Foutch 1985, Groot et al 1990, Kim \& lannotti 1999), but a detailed methodology has not been described, and biocompatibility towards the specific microorganism remains sometimes unexplored. Moreover, inconsistent results and relatively high variability is evidenced from different sources (Offeman et al 2005). 
The first systematic methodology for selecting a suitable solvent for extractive fermentation was proposed by Kollerup \& Daugulis $(1985,1986)$. The authors elaborated an Extractant Screening Program able to read a database of more than 1500 organic solvents, and to estimate their extraction capacities by means of UNIFAC coefficients. More sophisticated mathematical tools based on Computer Aided Molecular Design were also used in the last decade (Cheng \& Wang 2010, Wang \& Achenie 2002) to improve the search methods. Among the general criteria, extraction capacity towards the product to be recovered maybe considered the most important factor. It is often evaluated by the partition (or distribution) coefficient and product selectivity (related to water). In addition of high product partitioning, a good solvent must have no toxicity towards microorganisms. Solvent toxicity is correlated with their hydrophobic character (less water affinity implies more biocompatibility), which is commonly expressed as logP value (or log $\mathrm{K}_{\text {octanol-water }}$ ). However, a high hydrophobic behavior is inversely proportional to its extraction capacity. Therefore, the solvent selection for a extractive fermentation remains a complex task and compromise must be achieved between extraction capacity and biocompatibility. The search of an ideal solvent for the ABE extractive fermentation has been the subject of many works carried out from the early eighties and oleyl alcohol has been reported by several authors to be one of the most performing butanol extracting agents in $A B E$ fermentation. The major advantages are its non-toxicity towards Clostridium microorganisms and its acceptable distribution coefficient for butanol.

In this work, a solvent screening methodology leading to the search for high performance solvents to be used in a liquid-liquid extraction coupled to the ABE fermentation is proposed. Potential solvents belonging to different chemical families (alcohols, esters, oils and ionic liquids) were first identified in a pre-screening step based on data obtained from literature. This step allowed the creation of a homogenous database containing separation parameters and the selection of the candidate solvents. Then, few selected solvents from the prescreening step were tested experimentally for determining their (a) partition coeficients and selectivity and (b) their biocompatibility towards Clostridium acetobutylicum in a series of experiments based on microfermentations. In order to mimic a real system, culture broth obtained from a $2 \mathrm{~L}$ bioreactor fermentation with $C$. acetobutylicum was used as aqueous 
phase. Glucose consumption and total butanol production (in aqueous and organic phases) were compared to the results obtained in control fermentation (no solvent).

\section{Materials and Methods}

\section{Extractants}

The selected solvents from each chemical group were five alcohols (2-ethyl-1-hexanol, 1dodecanol, 2-butyl-1-octanol, 2-hexyl-1-decanol and oleyl alcohol), five esters (diisobutyl adipate, dibutyl phtalate, tributyl citrate, dibutyl sebacate and bis (2-ethylhexyl) adipate), four oils (silicon, sunflower, pomace and castor oils) and two water-immiscible ionic liquids (methyltrioctylammonium (Aliquat) chloride and trihexyl (tetradecyl) phosphonium chloride). Their characteristics are included as Additional Information (File A).

\section{Microorganism and culture media}

Clostridium acetobutylicum ATCC 824 was used as the solvent producing microorganism. All experiments were started with a frozen spore suspension in saline solution. $C$. acetobutilycum spores $(150 \mu \mathrm{L})$ were heated-schocked for $1 \mathrm{~min}$ at $100 \mathrm{oC}$ in order to induce spore germination and then they were used to inoculate $10 \mathrm{ml}$ of potato/glucose preculture medium, which was incubated anaerobically for $72 \mathrm{~h}$ at $360 \mathrm{C}$. The potato/glucose preculture medium contained per L: $250 \mathrm{~g}$ potato, $2 \mathrm{~g}\left(\mathrm{NH}_{4}\right)_{2} \mathrm{So}_{4}, 2 \mathrm{~g} \mathrm{CaCO}_{3}$ and $10 \mathrm{~g}$ glucose.

The pre-culture medium was transferred to $100 \mathrm{ml}$ of culture medium disposed in a $250-\mathrm{ml}$ sealed flask, which was previously purged with $\mathrm{N}_{2}$. The culture medium composition per $\mathrm{L}$

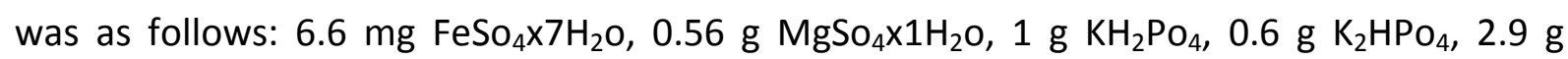
$\mathrm{CH}_{3} \mathrm{CooNH}_{4}, 0.1 \mathrm{~g} \mathrm{p}$-aminobenzoic acid, $2.5 \mathrm{~g}$ yeast extract and $60 \mathrm{~g}$ glucose. The flask was incubated at $360 \mathrm{C}$ in a shaker with gentle agitation.

In the case of microfermentations for biocompatibility evaluation, 12-ml tubes filled with 4 $\mathrm{ml}$ of culture medium were inoculated with cells growing at a high rate in a sealed bottle. The initial glucose concentration was fixed at $90 \mathrm{~g} / \mathrm{l}$.

To inoculate the 2-L bioreactor, the culture medium growing in sealed bottle was transferred to the reactor vessel after $24-36 \mathrm{~h}$ of incubation, when the culture is at maximal growth rate and before the cells start to sporulate. All of the culture media and material were previously autoclaved.

\section{Determination of partition coefficient and selectivity}


The extraction performance of each solvent was evaluated taking into account the partition coefficient (Kp) and the selectivity (Sel). The partition coefficient was calculated according to Eq. 1, where $[\mathrm{BuoH}]_{\text {org }}$ and $[\mathrm{BuoH}]_{\mathrm{aq}}$ are the equilibrium butanol concentrations in the organic and the aqueous phase, respectively.

$$
K_{B u O H}=\frac{[\mathrm{BuOH}]_{\text {rg }}}{[\mathrm{BuOH}]_{a q}} \text { Eq.1 }
$$

The selectivity (or separation factor) is expressed as the division of the distribution coefficient of that compound by the water distribution coefficient:

$$
\text { Sel }=\frac{K_{B u O H}}{K_{\text {water }}} \text { Eq. } 2
$$

The extractions were performed in $1.43 \times 12 \mathrm{~cm}$ conical graduated polyethylene (PE) tubes, with an aqueous to organic phase volume ratio of $2: 1$, and total volume of $7.5 \mathrm{ml}$. The tubes were shaken vigorously in horizontal position to completely emulsify both organic and aqueous phases at $360 \mathrm{C}$ for $2 \mathrm{~h}$. Then, the tubes were allowed to settle vertically for $24 \mathrm{~h}$.

In order to clear both phases and to break wall droplets or emulsions, the tubes were centrifuged during $20 \mathrm{~min}$ at $5000 \mathrm{rpm}$. Then, aliquots of the organic and aqueous phases were removed and stored for further analyses.

The organic phase concentration for butanol and other metabolites was estimated by mass balance, as expressed in Eq. 3

$$
\begin{gathered}
{[\mathrm{BuOH}]_{0} V_{0}=[\mathrm{BuOH}]_{a q} \sqcap V_{a q} \square[\mathrm{BuOH}]_{\text {org }} V_{\text {org }} \quad \text { Eq. } 3} \\
\frac{[\mathrm{BuOH}]_{b}}{[\mathrm{BuOH}]_{a q}} V_{0}=V_{a q}+K_{\mathrm{BuOH}} V_{\text {org }} \quad \text { Eq. } 4
\end{gathered}
$$

The performance of the extraction was evaluated by analytical quantification of final aqueous products (butanol, acetone, ethanol and acids). It was assumed that the solubility of the solvent is negligible. Taking this into account, the water concentration in aqueous phase was estimated using Eq.5

$$
\left[\mathrm{H}_{2} \mathrm{O}\right]_{q q}=1-\sum_{i}\left[\mathrm{C}_{i}\right]_{q q} \quad \text { Eq. } 5
$$


where i: 1 to number of compounds in the aqueous broth (butanol, acetone, ethanol, acids , glucose...). Standard deviations (SD) of both parameters were estimated from results of triplicate extraction runs.

The water content of the organic phase was evaluated by Karl-Fischer titration (Metrohm $795 \mathrm{KF})$.

\section{Biocompatibility evaluation}

Solvent toxicity towards $C$. acetobutylicum was evaluated by comparing glucose uptake and total butanol production between control and solvent-exposed microfermentations carried out by duplicate. Tests were conducted in $12 \mathrm{ml}$ PE tubes, with a ratio aqueous to organic phase equal to $1: 1$, and a total volume of $8 \mathrm{ml}$.

The tubes, with $4 \mathrm{ml}$ of growth broth, and the same volume of extractant were placed in anaerobic jar for $24 \mathrm{~h}$ previously to inoculation. All the tubes were inoculated at the same time at $10 \%(v / v)$ with the same fermentation culture. The microfermentation tubes were put again vertically in the anaerobic jar without agitation and were incubated at $360 \mathrm{C}$ during $72 \mathrm{~h}$. Then, the tubes were centrifuged during $20 \mathrm{~min}$ at $5000 \mathrm{rpm}$ and both phases were separated for further analysis.

\section{Analytical procedures}

Aqueous and organic phase compositions were determined by gas chromatography (GC) (HP6890 GC) with $\mathrm{N}_{2}$ as the carrier gas and a flame ionization detector. The GC was equipped with an Agilent 122-7032E column. The oven was kept isothermally at $70 \circ \mathrm{C}$ for $3 \mathrm{~min}$ and thereafter, temperature was increased up to $200 \mathrm{oC}$ at a gradient of $50 \mathrm{oC} / \mathrm{min}$.

To estimate the water content in aqueous phase, it was assumed that the aqueous solubility of the solvent was negligible. In the case of organic samples, hexane was combined at a ratio of $1: 1 \mathrm{vol}$, to the organic sample being analyzed. Only butanol was quantified in the organic phase at the end of microfermentations for biocompatibility evaluation. Quantification of glucose was performed using an enzymatic kit (Glucose-TR GoD-PoD, Ref.1001190, Spinreact, GI, Spain).

\section{Statistical analysis.}

Statistical analysis was conducted using the software R (R Development Core Team, 2009) to verify the existence of significant differences and to carry out control-to-solvent and pair- 
wise multiple comparisons. First, a one-way analysis of variance using parametric (ANOVA, based on a linear model) and non-parametric (Kruskal-Wallis) methods was carried out to determine if the values obtained using different solvents or group of solvents were significantly different. Then, if a difference between the mean values was confirmed, a posthoc analysis was applied to determine the values for which that difference was significant, considering a level of significance of 0.05. Dunnett/Tukey non-parametric test (R-package nparcomp) was applied when the number of samples was different between groups and/or the assumption of homogeneity of variances was not met. In all other cases a Tukey-HSD test was carried out.

\section{Results and discussion}

\section{Pre-screening step}

An initial list of 63 potential solvents was developed according to bibliographic research on extractive $A B E$ fermentation and equilibrium data (see Additional information - Appendix DB1). This database regroups the different chemical families that present affinity for butanol in an aqueous system, and compile the results obtained with different solvents already tested in $A B E$ or ethanol extractive fermentations. Qualitative or quantitative information including cost, handling properties, density, viscosity and other properties was also considered in the pre-screening. Even if these data are intrinsically heterogeneous, since the experimental procedure changes for each research work, some general trends are evidenced from the compiled data.

The selected indicator for biocompatibility assessment was the $\log \mathrm{P}(\log \mathrm{Kow})$. When this value was not available in literature, it was estimated by using the Rekker's group contribution method (Rekker 1977). It has been stated that solvents with a logP value lower than 4 are, in general, toxic to C. acetobutilycum (Kollerup \& Daugulis 1986). A solvent with a $\log P$ higher than 6 would preserve bacterial bioactivity, while for values between 4 and 6 it is difficult to conclude. As selection criterium, in this work it was decided to select solvents presenting $\log \mathrm{P}$ values equal or higher than 3, which were defined as 'a priory biocompatible' solvents.

For potential biocompatible solvents presenting acceptable affinity for ABE fermentation products (in terms of $\mathrm{K}$ and selectivity values), other important characteristics were 
examined: density difference with water, solubility, viscosity, etc. Solvents presenting clear unacceptable properties were discarded, and the best ranked solvents of each chemical family were selected for further experimental screening. However, if a solvent presents outstanding extraction performance parameters, but no biocompatibility a priori, it could be selected to be tested in a mixture with a poorer, but biocompatible, solvent.

As a general rule, alcohols presented the best extractability for butanol in terms of the highest distribution coefficient (K), which range from 3 to 8 . $K$ values for butanol and ethanol decrease as the concentration of hydroxyl groups decreases, which occurs when alkyl chain length of the solvent increases. Selectivity presented higher variability due to intrinsic larger measure errors. It was reported that selectivity for ethanol is affected by the level of branching of the molecule and/or the $\mathrm{OH}$ position (Offeman \& Stephenson 2008). For the case of butanol, selectivity substantially increased if the solvent alcohol is beta branched or the hydroxyl group is placed in the middle of the molecule.

Esters and ketones go second in terms of distribution coefficient for butanol. Ketones mix well with water and mutual solubility is more important than for the other chemical families. Thus, it is expected that important amounts of water would be absorbed into the ketone (organic) phase during the liquid-liquid extraction of aqueous ABE broth. Their higher solubility in water implies also less biocompatibility a priori.

The vegetable oils are presented in a different chemical family, even when these triglycerides extracted from plants can be considered fatty esters. There are few data related to the extraction capacity of these compounds towards alcohol, but some authors reported the non toxicity of vegetable oils towards Clostridium (Groot et al 1990). From the oils presented in the database, castor oil presents the highest distribution coefficient for butanol $(3.6 \mathrm{~g} / \mathrm{g})$ while the rest of the oils present distribution coefficients lower than 1 . Other criteria such as viscosity - and then energy requirement for stirring and pumping - must also be taken into account when dealing with oils as potential extractants.

Alkanes present the lowest distribution coefficient for butanol, acetone and ethanol, and do not appear suitable for the liquid-liquid extraction of the fermentation products. However, their use in solvent mixtures could be justified for improving other important criteria (e.g. viscosity). 
Other solvents such as halogenated hydrocarbons were reported to enhance the ethanol and acetone extraction, but not so much butanol (Dadgar \& Foutch 1985). However, their highly toxic character does not make them suitable for any extractive fermentation.

After applying the pre-screening, sixteen chemicals were selected to be experimentally evaluated for their extraction capacity in $A B E$ fermentation broth. These include five alcohols, five esters, four oils and two ionic liquids.

\section{Distribution coefficients and selectivity values}

The composition of $A B E$ fermentation broth may influence the solute mass transfer rate and the equlibrium data. It has been reported the production of high titers of a biosurfactant (bacteriocin-like autolysin) at the end of the exponential growth phase (Webster et al 1981). These compounds could reduce the extraction efficacy up to 10-15\% (Pursell et al 2004). Taking into account the effect of the culture broth composition, in this study the determination of the extraction performance was carried out using as aqueous phase a culture broth obtained from an ABE fermentation with $C$. acetobutylicum. Thus, the initial butanol, acetone and ethanol concentrations in the aqueous phase were 10.62, 5.43 and $1.52 \mathrm{~g} / \mathrm{L}$, respectively.

The values for butanol selectivity over water and butanol distribution coefficients obtained experimentally for the selected solvents are given in Table 1. The complete set of results concerning equilibrium partition coefficient for intermediates (acids) and co-products (acetone, ethanol) of the $A B E$ fermentation, and their selectivity values are presented as Additional Material (File B).

Distribution coefficients range from 0.4 to 12 and compare reasonably well with data available in literature. Highest distributions coefficients were found for the ionic liquids $\left(p<1 \times 10^{-7}\right.$, Tukey non-parametric comparison between mean values of solvent groups) and the lowest values correspond to oils and esters $(p=0.245)$. In general, the chemical families tested can be ranked as follows: ionic liquids > alcohols > ester oils.

Considering selectivity, silicon oil presented the highest value (Sel > 3000). This mineral oil consists in a polymerized siloxane, whose structure contains silicon, oxygen and an alkane side chain, which confers strong hydrophobic properties. On the other side, ionic liquids, two high density esters (dibutyl phthalate and tributyl citrate) and one alcohol (dodecanol) 
presented the lowest separation factors $(p>0.187$ between them, and $p<0.0052$ compared with the other solvents, Tukey-HSD). Few data for selectivity values is available in literature. Some trends are revealed when selectivity is plotted against distribution coefficients for the oils, esters and alcohols datasets separately. Figure 1.A displays butanol extraction results obtained for alcohols, which are one of the better classes of solvent in terms of distribution coefficient. 2-ethyl-1-hexanol presents the highest partition coefficient. However, it cannot be considered as a 'biocompatible a priori' solvent $(\log \mathrm{P}<3)$. It is proposed at this step as a potential solvent to be used in mixtures.

Oley alcohol is the most studied solvent for in situ ABE extractive fermentations. Values of $\mathrm{K}_{\mathrm{BuoH}}$ were reported as 3.2 (Evans \& Wang 1988), 4 (Malinowski \& Daugulis 1994) and 3.3 (Ishizaki et al 1999). Variation between these values and that reported in the present study (4.5) may be due to differences in solvent purity, operating conditions, aqueous broth composition or analytical methods employed.

Influence of the branching degree is observed when comparing the selectivity of the isomers 1-dodecanol and 2-butyl-1-octanol (171 and 645, respectively). Increase of selectivity by location of the hydroxyl group in the middle of the molecule has already been reported for ethanol liquid-liquid extraction (Offeman \& Stephenson 2008). Other important advantage of branched high molecular weight alcohols is that they are liquid at room temperature, whereas the unbranched $n$-alcohols are solid.

Distribution coefficients of esters (Figure 1.B) fall within a relatively narrow range, and are significantly lower than those obtained for alcohols $\left(p<1 \times 10^{-5}\right.$, Kruskal-Wallis). Dibutyl phtalate and tributyl citrate present the lowest value for selectivity $(p=0.753$ between them, $\mathrm{p}<0.0025$, when compared with the other esters, Tukey-HSD between esters). In addition, these are the only extractants whose density is higher than that of water. This phase inversion may difficult the operation at higher scale.

Considering the adipate and sebacate based esters, some correlation appears again between branching degree and selectivity. In fact, dibutyl sebacate, which presents the lowest selectivity of the three compounds, is the only one having no branching in both side chains.

Three vegetable oils and one mineral oil were tested (Figure 1.C). Castor oil presents the highest $\mathrm{K}_{\mathrm{BuOH}}(2.6)$ vs the others oil tested $\left(\mathrm{p}<2 \times 10^{-5}\right.$, Tukey-HSD between oils). However, low 
selectivity and high viscosity (1500 cP) makes the use of this oil in commercial purposes less attractive. Viscosities of the other three oils are in the range 10-85 cP.

High butanol affinity in castor oil can be explained by its predominant fatty acid structure which is the ricinoleic acid. It has an additional 12-hydroxyl group, conferring to the molecule supplementary hydrogen bonding site. This gives also affinity for water, explaining its lower selectivity.

Pomace and sunflower oil form a group with a $\mathrm{K}$ coefficient near to 0.5 and selectivity around 600 ( $\mathrm{p}$ for K and Sel were 0.798 and 0.916, respectively, Tukey-HSD between oils). These similar performances are due to their common dominant fatty acid structure: linoleic acid (double unsaturated, higher proportion in sunflower oil) and oleic acid (single saturated, higher proportion in olive oil).

Silicon oil presents the highest value for selectivity of all the solvents tested due to its strongly hydrophobic chemical structure (siloxane polymer).

Results obtained with the ionic liquids are depicted in Figure 1.D. The highest distribution coefficients ( $p<2 \times 10^{-4}$, Dunnett's non-parametric comparison between solvent groups) were obtained compared to the other solvents. $\mathrm{K}_{\mathrm{BuOH}}$ values are approximately 3-4 times higher than that obtained for oleyl alcohol. Lowest selectivities were also obtained by ionic liquids, and were similar to that obtained with esters $(p=0.178)$, but significantly higher than those of alcohols and oils $\left(\mathrm{p}<1 \times 10^{-6}\right.$, Dunnett's non-parametric comparison between solvent groups).

The two ionic liquids present an extremely high viscosity ( $1800 \mathrm{cP}$ at $\left.25^{\circ} \mathrm{C}\right)$ which could represent an important drawback to their use for an in situ extractive configuration. Other important characteristic was their ability to form emulsions. They may exhibit surfactant-like behavior and, at the end of each extraction run, an intermediary 'new' apparent phase appeared. The formation of stable association of solvent molecules (clusters) at the solvent/aqueous interface might difficult the operation and entrain some quantitative solvent losses.

For the purpose of limiting the set of potential solvents, and based on extraction performance characteristics and other observed parameters during the extraction runs (viscosity, emulsion formation, etc), two alcohols (oleyl alcohol and 2-butyl-1-octanol), two 
esters (diisobutyl adipate and bis (2-ethyl-1-hexyl) adipate) and three oils (pomace, sunflower and silicon oils) were selected to be further screened for their biocompatibility towards C. acetobutylicum.

In addition, a mixture containing 20/80 (v/v) of 2-ethyl-1-hexanol and pomace oil, respectively, was also investigated. The objective was to determine whether this mixture could improve the overall distribution coefficient of the vegetable oil having poor affinity for butanol $(K=0.4)$, keeping low aqueous concentration of the toxic solvent.

From an engineering perspective, an optimal balance between $\mathrm{K}_{\mathrm{BuOH}}$ and selectivity will depend on process configuration (regeneration mode) and final product specifications. Operational cost generated by energy demand may be important in ABE extractive fermentation system given the latent heat of vaporization of water $(>2400 \mathrm{~kJ} / \mathrm{kg}$ ) relative to that of butanol $\left(\sim 700 \mathrm{~kJ} / \mathrm{kg}\right.$ ), and their respective boiling points (100 and $118{ }^{\circ} \mathrm{C}$, respectively). On the other hand, 'solvent to feed' ratio is an important factor that affects capital expenditure and size of the equipment.

\section{Biocompatibility tests in microfermentations}

Whether in situ or in stream configuration system, the contact between the solvent and the microorganism is unavoidable in an extractive fermentation. Therefore, biocompatibility towards the specific biocatalyst must be guaranteed in such a system. An extended explanation about the mechanism of different types of toxicity (dissolved or biphasic toxicity), their effects on cell's membrane, and potential ways to predict the biocompatibility a priori can be found in previous works (Vermue \& Tramper 1995, Salter \& Kell 1995). In this study, biocompatibility evaluation of seven solvents and one solvents mixture was experimentally determined in microfermentations.

Glucose consumption is presented in Figure 2. It is observed that all the solvents, except 2ethyl-1-hexanol $(2 \mathrm{E} 1 \mathrm{H})$ and the mixture with $2 \mathrm{E} 1 \mathrm{H}$, are biocompatible. Glucose consumption of the mixture containing $20 \%(\mathrm{v} / \mathrm{v})$ of 2-ethyl-1-hexanol in pomace oil was similar to that of the negative control (with 2-ethyl-1-hexanol) ( $p=0.998$, ANOVA). This means that a concentration as low as $20 \%$ of 2-ethyl-1-hexanol (toxic solvent) towards pomace oil (biocompatible matrix) would be enough to result in a non-biocompatible mixture. 
Standard deviations (SD) of glucose consumption for solvent-free control and for all microfermentations were lower than 3, except for dodecanol and diisobutyl adipate (12 and 30, respectively). Dodecanol has already been reported to be toxic towards $C$. acetobutylicum (Evans \& Wang 1988), and some inconsistency with the use of this solvent was also highlighted for ethanol extractive fermentation (Offeman \& Stephenson 2008).

In this work, logP values of diisobutyl adipate and dodecanol (4.19 and 4.99, respectively) are into the intermediary region, near the 'biological threshold'. Glucose consumption for these solvents was similar to that of the control fermentation ( $p>0.999$, Dunnett's nonparametric control-to-solvent comparison). Nevertheless, due to the high SD, these compounds will not be considered. The other solvent falling in the logP critical zone, 2-butyl1-octanol, with a logP of 4.99, resulted to be biocompatible. In Figure 3, both parameters, $\log \mathrm{P}$ and glucose consumption, are represented. $\log \mathrm{P}$ is inversely correlated with alcohol distribution coefficient, and consequently, it should be convenient to work as near as possible to the 'biological threshold' of the microorganism.

Another important factor to be considered is the agitation rate. Increased agitation may shift the 'critical threshold' to the right, increasing the presence of toxic solvent, as result of better transfer of the solvent into the aqueous phase. A compromise must be achieved between mass transfer and bioactivity preservation.

In the biocompatibility tests, neither the geometry of the tubes, with a high L/D ratio, nor the gentle agitation of the anaerobic jar, which kept the interface completely unbroken, favoured mass transfer. However, at the end of the microfermentations, equilibrium conditions were attained. This can be observed in Figure 4, in which a comparison between experimental data (total butanol produced in both phases) and theoretical equilibrium partition for butanol (using $\mathrm{K}_{\mathrm{BuOH}}$ estimated from the extraction runs) is presented.

Figure 5 displays glucose consumption and butanol production over the control fermentation for the biocompatible solvents. It is observed that the excess of glucose consumption over solvent free fermentation does not lead systematically to the same amount of overproduction of butanol. In fact, oleyl alcohol and 2-butyl-1-octanol present the highest final yields for butanol $\left(\mathrm{Y}_{\mathrm{BuOH} / \mathrm{Glu}}\right.$, in \%w/w) $\left(25.50\right.$ and 27.43 , respectively, $\left.\mathrm{p}<8 \times 10^{-5}\right)$, while silicon oil presents the lowest yield $\left(16.45, p<8 \times 10^{-4}\right)$. Adipate ester and vegetable oils 
resulted in similar yields ( 21.03 and $20.05-20.51$, respectively, with $0.707<p<0.987$ between them, Tukey-HSD).

These results may indicate some influence of the solvent on $A B E$ metabolism. Besides, $\mathrm{Y}_{\mathrm{BuOH} / \mathrm{Glu}}$ reveals correlation with butanol and butyric acid distribution coefficients between phases. Some hypotheses that could be formulated in order to explain theses results are the following:

a) The organic phase may act as a 'source' of butyric acid during the solventogenesis, modifying product yield structure. It has been reported that butyric acid addition during solventogenic phase enhances butanol production (Kollerup \& Daugulis 1986, Cheng \& Wang 2010, Wang \& Achenie 2002).

b) Acidic conditions favours solventogenic behaviour and butanol metabolic pathway is stimulated at low pH (Wang \& Achenie 2002). Butyric acid from organic phase would have created "NADH pressure" by lowering the $\mathrm{pH}$ after the metabolic switch. This redox imbalance would enhance product yield, since oxidation of NADH is coupled to butanol and ethanol production, but not to acetone.

c) Lower aqueous concentration of butyric acid during acidogenic step could have preserved to a certain extent the solventogenic role of the cells.

d) Increasing the efficacy of butanol removal from the aqueous phase could have improved specifically the butanol metabolic pathway by kinetics or thermodynamic means (or both).

However, in order to evaluate these hypotheses, more specific experimentation needs to be carried out. Detailed profiles of biphasic fermentations with different types of solvents appear necessary to understand the relation between solvent nature and $A B E$ metabolic pathways.

The results obtained from microfermentations to determine the degree of biocompatibility are compiled in Table 2. The extent to which a solvent is biocompatible or toxic towards a microorganism can be determined by measuring the percentage of surviving cells, cell density, substrate utilization or product formation. In this work, glucose uptake and butanol production were the criteria chosen for screening solvent biocompatibility. Other parameters, like cell survival or cell viability are important criteria in mid-long term fermentations and should be considered in further steps of solvent selection. Indeed, if 
metabolic activity is maintained but low cell survival is observed, this can be explained by an initial harmless solvent action, when intracellular solvent concentration is not high enough yet. With time, the accumulation of solvent molecules could accelerate enzyme denaturation and eventually lead cell to death.

\section{Conclusions}

Sixteen chemicals were experimentally evaluated for their potential use in ABE extractive fermentation. Two mass transfer parameters (distribution coefficient and selectivity of the ABE products on the extractant solvents) and two parameters related to biocompatibility (glucose consumption and total butanol production) were determined.

Highest distribution coefficients were found for ionic liquids and the lowest values for oils. In general, the solvents tested can be ranked as follows in terms of distribution coefficient: IL >> alcohols > esters oils. Concerning selectivity, silicon oil presented the highest value ( 3000), whereas ionic liquids co-extracted important amounts of water and presented the lowest selectivities $(<80)$.

It was observed that increasing glucose consumption for biocompatible solvents, with respect to non-solvent fermentations, does not lead systematically to overproduction of butanol. This fact reveals some influence of the solvent used in liquid extraction on $A B E$ metabolism.

The solvent 2-butyl-1-octanol presented the highest butanol yield $\left(\mathrm{Y}_{\mathrm{BuOH} / \mathrm{Glu}}\right)$ and it was also found (in this work) that it have good extraction characteristics. 2-Butyl-1-octanol belongs to the family of Guerbet alcohols and it is commonly used as raw material in the industries of cosmetics, drug delivery, metal processing, fiber finish, thermostable and biodegradable lubricant and solvent, as well as surfactant. However, it has not been extensively used in extractive $A B E$ fermentations, and for this reason a more detailed study need to be carried out to further evaluate its potential use in butanol production. 


\section{References}

Barton WE, Daugulis AJ (1992) Evaluation of solvents for extractive butanol fermentation with Clostridium acetobutylicum and the use of poly(propylene glycol) 1200. Appl. Microbiol. Biotechnol 36:632-639.

Cheng $\mathrm{H}$, Wang $\mathrm{F}$ (2010) Computer-aided biocompatible solvent design for an integrated extractive fermentation-separation process. Chem. Eng. Journal 162:809-820.

Dadgar AM, Foutch GL (1985) Evaluation of solvents for the recovery of Clostridium fermentation products by liquid-liquid extraction. Biotechnol. Bioeng. Symp 15:612-620.

Evans PJ, Wang HY (1988) Response of Clostridium Acetobutylicum to the presence of mixed extractants. Appl. Biochem. Biotechnol. 17:75-192.

Ezeji T, Milne C, Price ND, Blaschek HP (2010) Achievements and perspectives to overcome the poor solvent resistance in acetone and butanol-producing microorganisms. Appl. Microbiol. Biotechnol. 85:1697-1712

Groot WJ, Soedjak PB, van der Lans RGJM, Luyben KchAM (1990) Butanol recovery from fermentations by liquid-liquid extraction and membrane solvent extraction. Bioprocess Eng. 5:203-216.

Ishizaki Al, Michiwaki S, Crabbe E (1999) Extractive Acetone-Butanol-Ethanol fermentation using methylated crude palm oil as extractant in batch culture of Clostridium saccharoperbutylacetonicum NI-4 (ATCC 13564). J. Bioscience Bioeng. 3:352-356.

Jones D.T. Woods D.R. "Acetone-Butanol Fermentation Revisited". 1986. Microbiological Reviews. 50. 4. 484-524

Kim J, lannotti EL (1999) Extractive recovery of products from fermentation broths. Biotechnol. Bioprocess Eng. 4:1-11

Kollerup F, Daugulis AJ (1986) Ethanol production by extractive fermentation solvent identification and prototype development. Can. J. Chem. Eng. 64:598-606.

Kollerup F, Daugulis AJ (1985) Screening and indentification of extractive fermentation solvents using a database. Can. J. Chem. Eng. 63:919-927.

Kollmer A, Schmid A, Rudolf von Rohr Ph, Sonnleitner B (1999) on liquid-liquid mass transfer in two-liquid -phase fermentations. Biopr. Eng. 20:441-448.

Leland MV (2008) Separation technologies for the recovery and dehydration of alcohols from fermentation broths. Biofuels, Bioprod. Bioref. 2

Malinowski JJ, Daugulis AJ (1994) Salt effects in extraction of ethanol, 1-butanol and acetone from aqueous solutions. Aiche J. 40(9):1459-1465.

Offeman RD, Stephenson SK, Robertson GH, orts WJ (2005) Solvent extraction of ethanol from aqueous solutions. I. Screening methodology for solvents. Ind. Eng. Chem. Res. 44:6789-6796.

Offeman RD, Stephenson SK (2008) Extraction of ethanol with higher alcohol solvents and their toxicity to yeast. Sep. Purif. Technol. 63:444-451.

Oudshoorn A,van der Wielen LAM, Straathof AJJ (2009) Assesment of options for selective 1butanol recovery from aqueous solution. 2009. Ind. Eng. Chem. Res. 48:7325-7336. 
Pursell MR, Mendes-Tatsis MA, Stuckey DC (2004) Effects of fermentation broth and biosurfactants on mass transfer during liquid-liquid extraction. Biotechnol. Bioeng. 2:155-165.

Qhreshi N, Hughes S, Maddox IS, Cotta MA (2005) Energy-efficient recovery of butanol from model solutions and fermentation broth by adsorption. Bioprocess Biosyst. Eng. 27:215222.

R Development Core Team (2009) R: A Language and Environment for Statistical Computing. R Foundation for Statistical Computing (http://www.R-project.org), Vienna, Austria, ISBN 3-900051-07-0.

Rekker RF (1977) The hydrophobic fragmental constant. Elsevier Scientific Pub. Co., New York.

Salter GJ, Kell DB (1995) Solvent selection for whole cell biotransformations in organic media. Crit. Rev. Biotechnol. 15:(2)3-77.

Vermue $\mathrm{MH}$, Tramper J (1995) Biocatalysis in non-conventional media: Medium engineering aspects. Pure Appl. Chem. 67(2):345-373.

Wang Y, Achenie $L$ (2002) Computer-aided solvent design for extractive fermentation. Fluid Phase Equilib. 201:1-18.

Webster JR, Reind SJ, Jones DT, Woods DR (1981) Purification and characterization of an autolysin from Clostridium acetobutylicum. Appl. Env. Microbiol. 41(2):371-374.

Woodley JM, Bisschops M, Straathof AJJ, ottens M (2008) Future directions for in-situ product removal (ISPR). J. Chem. Technol. Biotechnol. 83:121-123. 


\section{Tables}

Table 1. Extraction results at $36^{\circ} \mathrm{C}$ and $[\mathrm{BuoH}]=10.6 \mathrm{~g} / \mathrm{l}$. and comparison with literature data

\begin{tabular}{|c|c|c|c|c|c|c|}
\hline \multirow[b]{2}{*}{ Solvents } & \multicolumn{2}{|c|}{ This work } & \multicolumn{4}{|c|}{ Literature } \\
\hline & $\mathrm{KBuOH}(\mathrm{SD})$ & Sel BuOH(SD) & $\begin{array}{c}\mathrm{K} \\
\mathrm{BuO} \\
\mathrm{H}\end{array}$ & $\begin{array}{c}\text { Sel } \\
\mathrm{BuO} \\
\mathrm{H}\end{array}$ & $\begin{array}{c}\mathrm{T}\left({ }^{\circ} \mathrm{C}\right) / \mathrm{BuOH} \\
\text { conc. }\end{array}$ & Reference \\
\hline \multicolumn{7}{|l|}{ Alcohols } \\
\hline 2-butyl-1-octanol & $6.76(0.25)$ & 644.8(23.5) & $\mathrm{N} / \mathrm{A}$ & $\mathrm{N} / \mathrm{A}$ & $\mathrm{N} / \mathrm{A}$ & --- \\
\hline \multirow[t]{3}{*}{ Oleyl alcohol } & $4.57(0.21)$ & 294.7(11.3) & 3.2 & $\mathrm{~N} / \mathrm{A}$ & $34 / 1.2 \% w t$ & Evans et al 1988 \\
\hline & $4.57(0.21)$ & 294.7(11.3) & 4 & $\mathrm{~N} / \mathrm{A}$ & $24 / 1.5 \% w t$ & Malinowski et al 1994 \\
\hline & $4.57(0.21)$ & 294.7(11.3) & 3.3 & $\mathrm{~N} / \mathrm{A}$ & $30 / 1.5 \% w t$ & Ishizaki et al 1999 \\
\hline 2-ethyl-1-hexanol & $7.95(1.52)$ & $311.1(61.6)$ & 6.1 & 276 & $28 / 2 \% \mathrm{vol}$ & Kim et al 1990 \\
\hline 2-hexyl-1-decanol & $3.41(0.27)$ & $509.2(36.8)$ & $\mathrm{N} / \mathrm{A}$ & $\mathrm{N} / \mathrm{A}$ & $\mathrm{N} / \mathrm{A}$ & --- \\
\hline \multirow[t]{2}{*}{ 1-dodecanol } & $5.06(0.32)$ & $171.5(7.1)$ & 5.1 & $\mathrm{~N} / \mathrm{A}$ & $34 / 1.2 \% w t$ & Evans et al 1988 \\
\hline & $5.06(0.32)$ & $171.5(7.1)$ & 6 & 140 & $25 / 1.5 \% w t$ & Groot et al 1990 \\
\hline
\end{tabular}

Esters

\begin{tabular}{l|cc|cccl}
\hline Diisobutyl adipate & $2.6(0.07)$ & $834.1(24.4)$ & 2.5 & 3 & $34 / 2 \% w t$ & Groot et al 1990 \\
\hline Dibutyl sebacate & $1.89(0.02)$ & $474.0(4.2)$ & 1.8 & $\mathrm{~N} / \mathrm{A}$ & $37 / 1 \%$ vol & Barton et al 1992 \\
\hline Dibutyl phtalate & $1.91(0.12)$ & $36.3(4.4)$ & 1.8 & $\mathrm{~N} / \mathrm{A}$ & $37 / 1 \% \mathrm{vol}$ & Barton et al 1992 \\
& $1.91(0.12)$ & $36.3(4.4)$ & 1.4 & 4 & $37 / 2 \% w t$ & Groot et al 1990 \\
\hline Bis(2E1H adipate) & $1.83(0.05)$ & $668.3(80.9)$ & 1.6 & 1.6 & $37 / 1 \%$ vol & Barton et al 1992 \\
\hline Tributylcitrate & $1.67(0.11)$ & $73.7(11.9)$ & 2.4 & $\mathrm{~N} / \mathrm{A}$ & $37 / 2 \% w t$ & Groot et al 1990 \\
\hline
\end{tabular}

Oils

\begin{tabular}{l|cc|cccc}
\hline SuN/Alower oil & $0.44(0.07)$ & $623.4(107.0)$ & N/A & N/A & N/A & -- \\
\hline Silicon oil & $0.59(0.04)$ & $3161.9(8.8)$ & N/A & N/A & N/A & --- \\
\hline Pomace oil & $0.62(0.12)$ & $577.6(124.7)$ & N/A & N/A & N/A & -- \\
\hline Castor oil & $2.85(0.46)$ & $285.7(60.3)$ & 2.6 & 270 & $37 / 2 \% w t$ & Groot et al 1990 \\
\hline
\end{tabular}

Ionic liquids

\begin{tabular}{l|cl|llll}
\hline Aliquat 336 & $8.86(0.91)$ & $41.7(3.8)$ & N/A & N/A & N/A & --- \\
\hline Phosphonium & $11.55(0.64)$ & $83.0(2.1)$ & N/A & N/A & N/A & --- \\
\hline
\end{tabular}


Table 2. Butanol extraction performance and biocompatibility results

\begin{tabular}{lccc}
\hline & \multicolumn{2}{c}{ BuOH extraction performance } & Biocompatibility \\
& K(S.D) & Selectivity(S.D) & \% $\Delta$ gluc.(S.D) \\
\hline Pomace oil & $0.63(0.12)$ & $577(124.7)$ & $12.8(0.6)$ \\
\hline Sunflower oil & $0.43(0.07)$ & $623(107)$ & $11.5(0.4)$ \\
\hline Oleyl alcohol & $4.57(0.21)$ & $295(11.3)$ & $13.2(3.4)$ \\
\hline 2-butyl-1-octanol & $6.76(0.25)$ & $644(23.6)$ & $7.0(2.6)$ \\
\hline Bis(2-ethyl-1-hexyl) adipate & $1.83(0.05)$ & $668(80.9)$ & $21.3(2.2)$ \\
\hline Silicon oil & $0.60(0.04)$ & $3161(8.85)$ & $16.2(0.4)$ \\
\hline
\end{tabular}

\section{Figures}

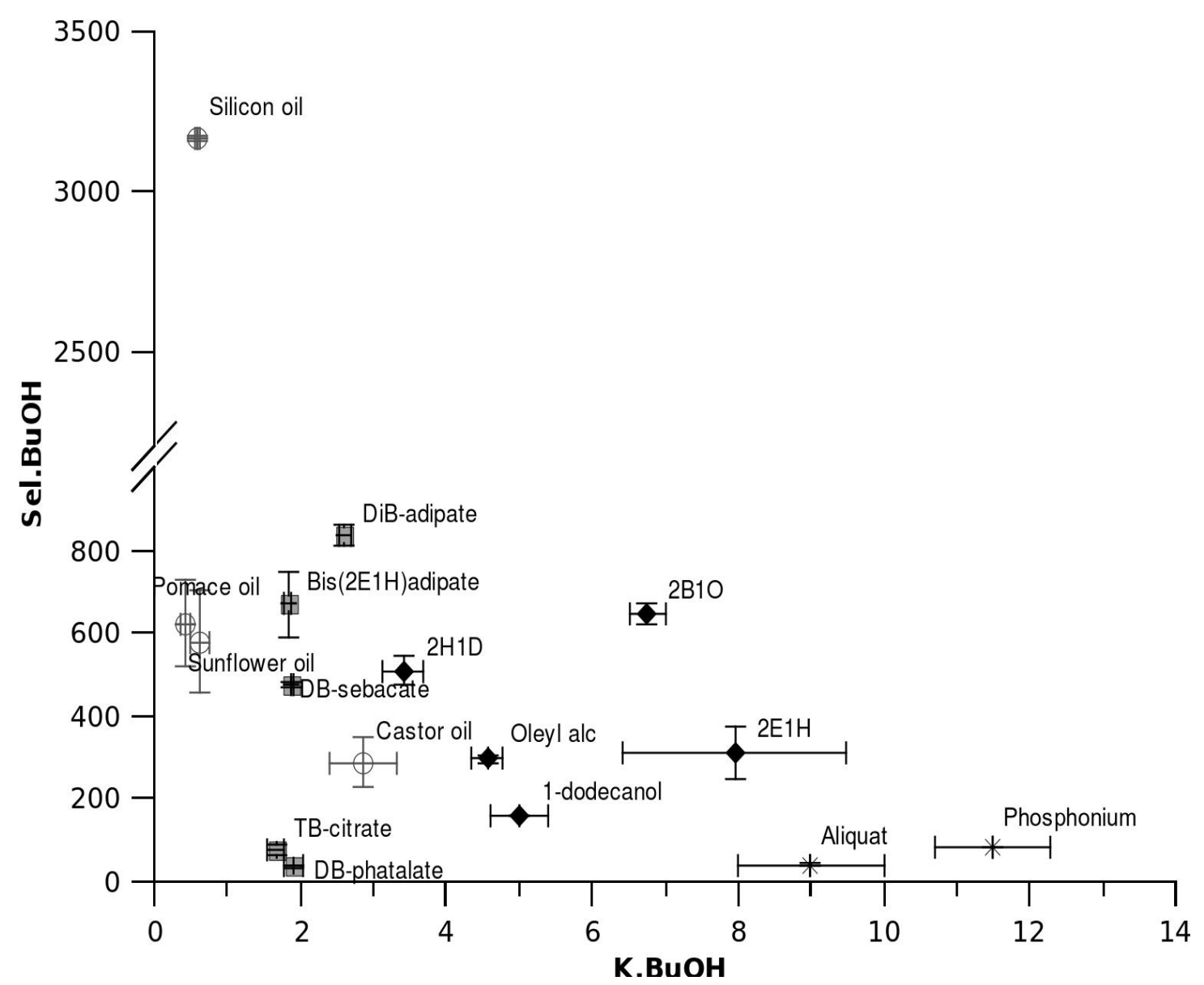

Figure 1. Distribution coefficients (K) and selectivity of butanol on $A$ ) five alcohols (black diamonds), B) five esters (gray squares), C) four oils (void circles) and D) two ionic liquids (black stars). 


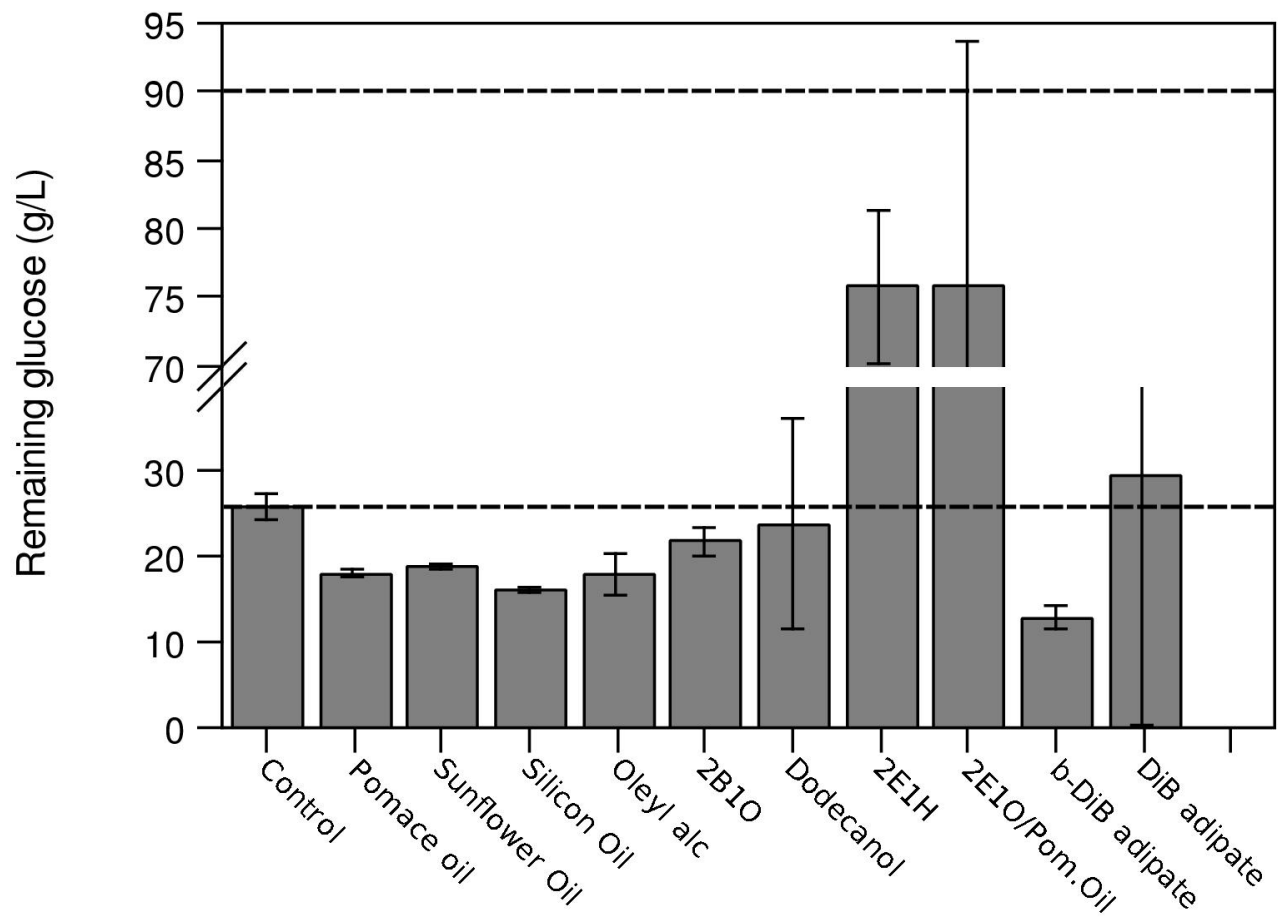

Figure 2. Remaining glucose in screening microfermentations

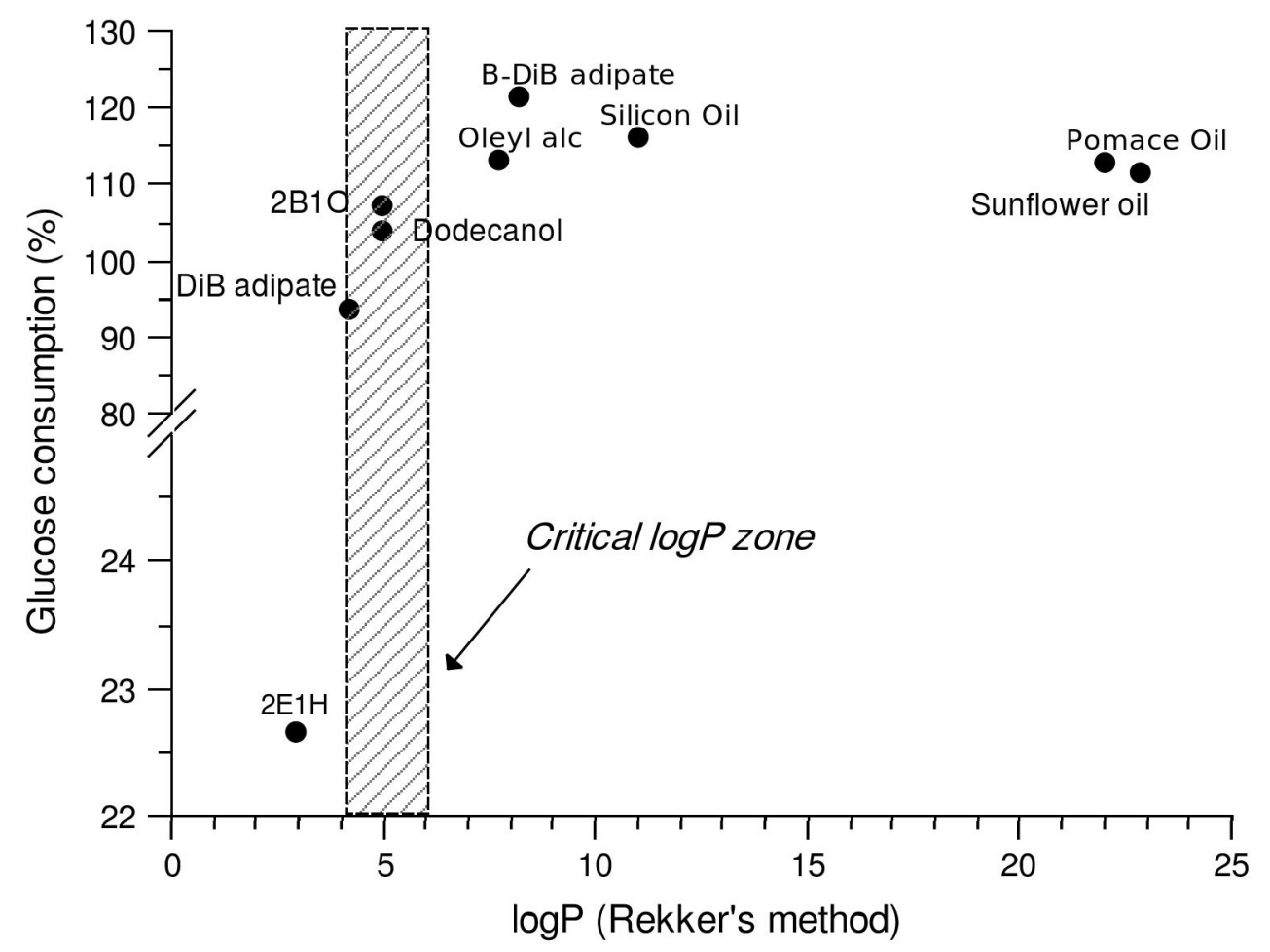

Figure 3. Glucose consumption over control fermentation vs estimated logP value of solvents investigated 


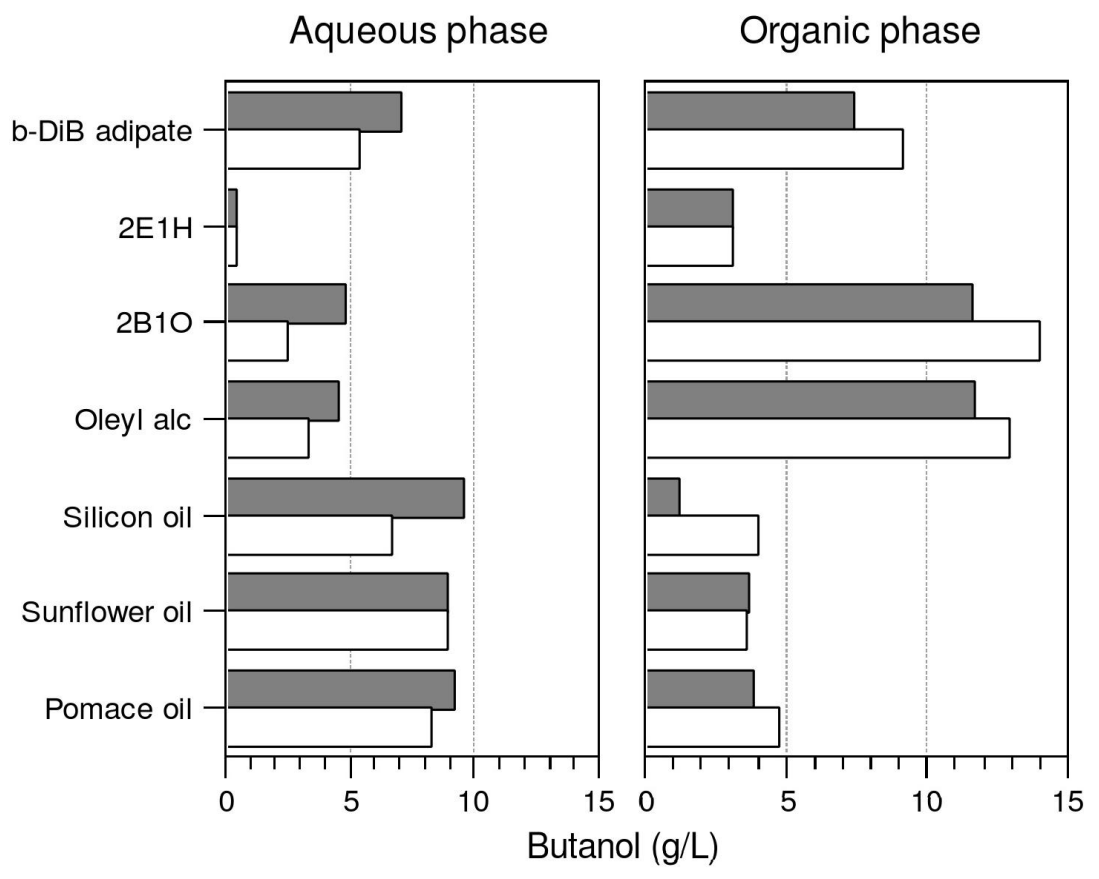

Figure 4. Partition of butanol into aqueous and organic phases (gray) comparison to equilibria data (white)

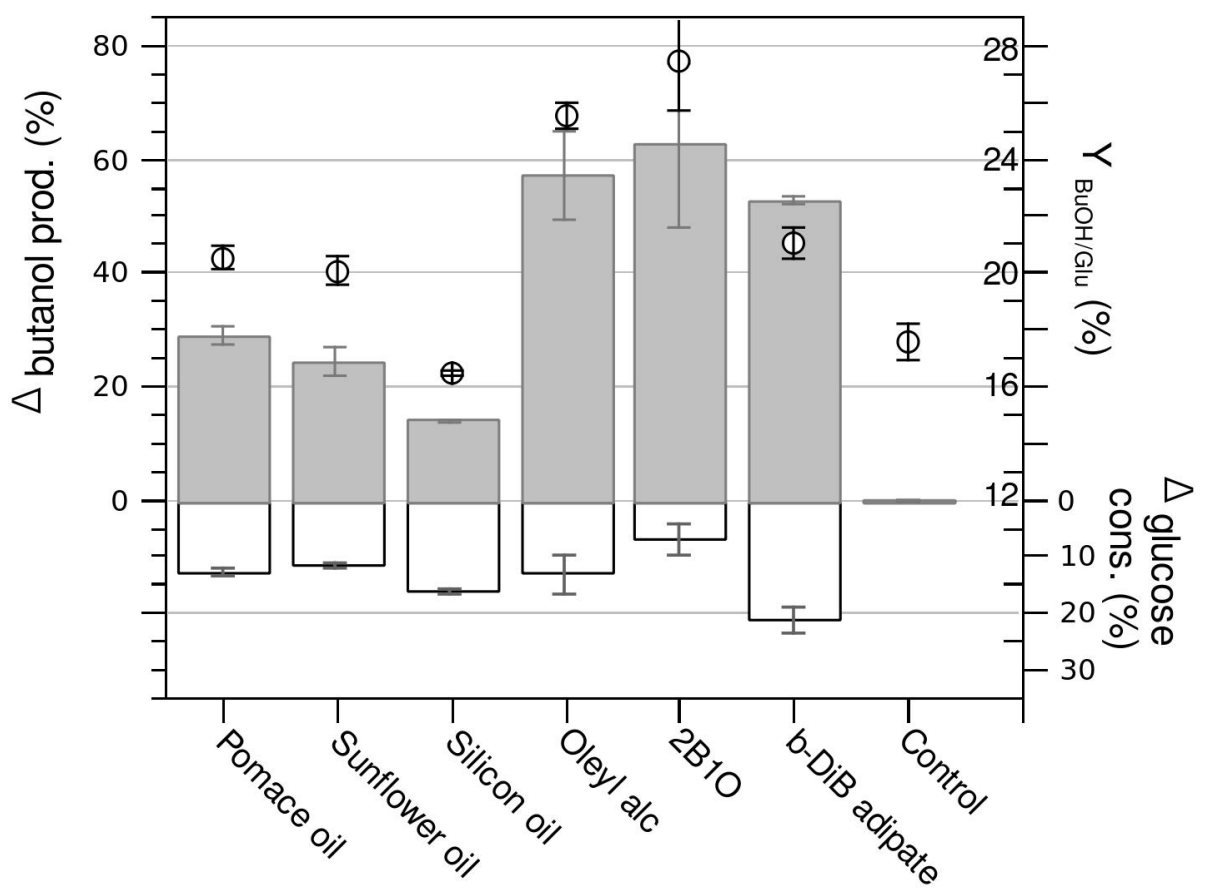

Figure 5. Increase of glucose consumption (white bars) and butanol production (gray bars) over control fermentation. Yield of butanol production with respect to consumed glucose is also shown (void circles) 